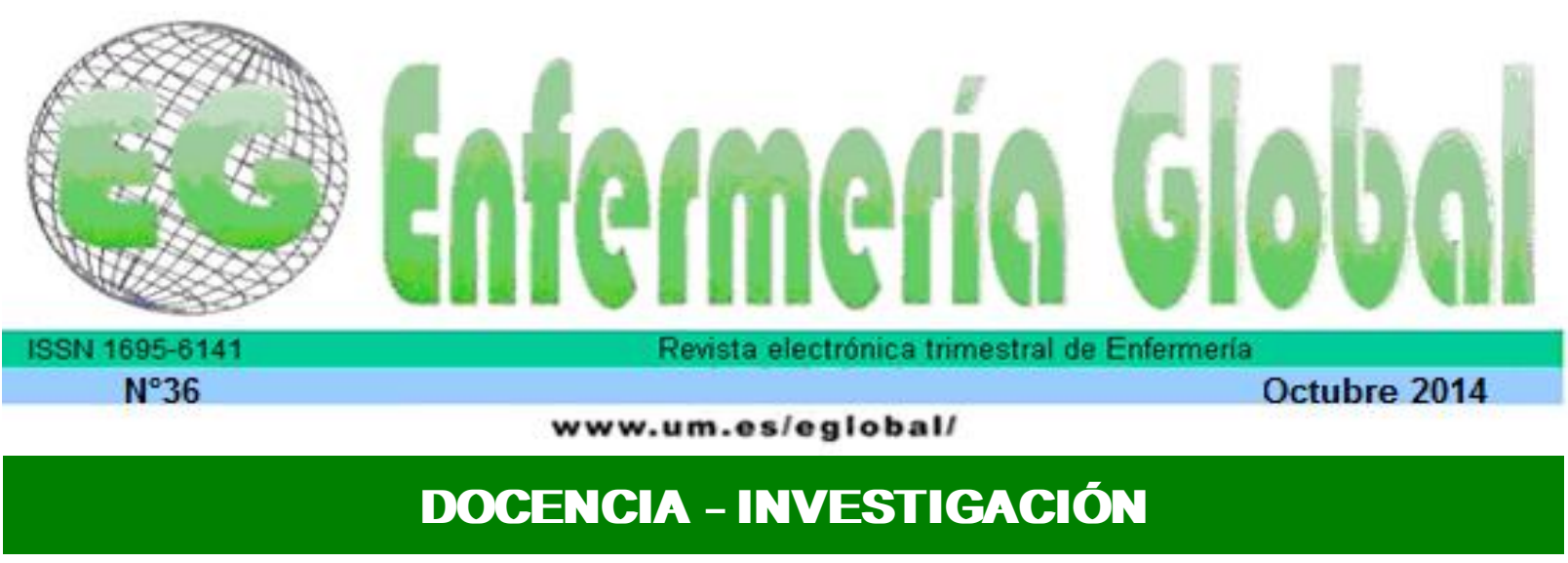

\title{
¿Es efectivo el aprendizaje sobre alfabetización en información para estudiantes de enfermería?
}

Is information literacy learning effective for nursing students?

\section{*Guerra Martín, María Dolores **Lima Serrano, Marta ***Zambrano Domínguez, Encarnación María ****Lima Rodríguez, Joaquín Salvador}

\begin{abstract}
*Diplomada en Enfermería, Licenciada en Antropología, Profesora Titular Departamento de Enfermería. Facultad de Enfermería, Fisioterapia y Podología. Universidad de Sevilla. **Diplomada en Enfermería, Licenciada en Antropología. Profesora Ayudante Doctora. Departamento de Enfermería. Facultad de

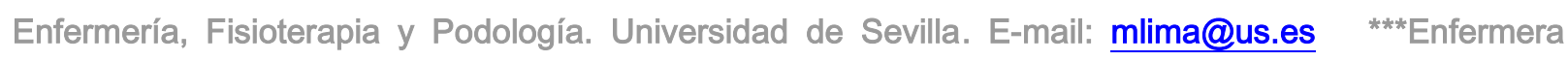
asistencial en el Hospital San Juan de Dios del Aljarafe. Sevilla. ${ }^{* * * *}$ Diplomado en Enfermería, Licenciado en Antropología y Doctor por la Universidad de Sevilla. Profesor Titular Departamento de Enfermería. Facultad de Enfermería, Fisioterapia y Podología. Universidad de Sevilla.
\end{abstract}

Palabras clave: Alfabetización en Información; Estudiantes de Enfermería; Procesos de Aprendizaje. Keywords: Information Literacy; Nursing Students; Learning Processes.

\section{RESUMEN}

Objetivo: Es urgente realizar programas dirigidos a las enfermeras de pregrado para desarrollar destrezas informáticas y sobre alfabetización de la información a largo plazo. Por ello se propuso mejorar las destrezas en alfabetización en información de los/as estudiantes de Enfermería y evaluar el proceso de aprendizaje.

Metodología: Se ha desarrollado un proceso enseñanza-aprendizaje para la alfabetización informacional de 80 estudiantes, que debían presentar para la evaluación un informe del proceso de aprendizaje. Se ha utilizado un diseño cuasiexperimental con un grupo para la evaluación de conocimientos adquiridos, al que se le aplicó un pretest y un postest.

Resultados: El $82.5 \%$ de los/as estudiantes ha superado el informe. Casi todos los participantes se han mostrado satisfechos/as con el proceso de aprendizaje. En la autovaloración de los conocimientos adquiridos, las medias obtenidas fueron significativamente mayores en el postest, siendo el tamaño de efecto muy grande.

Conclusiones: Existen evidencias de que el proceso enseñanza-aprendizaje para mejorar la alfabetización en información ha sido efectivo. La principal contribución ha sido incorporar la alfabetización de la información en el currículum de los/as estudiantes. 


\section{ABSTRACT}

Objective: The structured development on informatics and long term information literacy skills are urgently required for undergraduate nurses. Therefore, the improvement in the skills of information literacy of nursing students and evaluation of the learning process were proposed.

Methods: A teaching-learning process on information literacy was developed with 80 students, who had to submit a report for evaluation of the learning process. A quasi-experimental design was used with a group for the evaluation of acquired knowledge, to which a pretest and post-test was applied.

Results: $82.5 \%$ of students passed the report. Almost all participants were satisfied with the learning process. In the self-assessment of knowledge acquired, the means obtained were significantly higher in the post-test, while the effect size was very high.

Conclusions: There is evidence that the teaching-learning process to improve information literacy has been effective. The main contribution has been to incorporate information literacy into the students' curriculum.

\section{INTRODUCCIÓN}

Es urgente realizar programas dirigidos a las enfermeras de pregrado para desarrollar destrezas informáticas y sobre alfabetización de la información a largo plazo ${ }^{(1)}$. Las nuevas tecnologías de la información y comunicación (TIC) permiten el acceso de forma permanente a volúmenes crecientes de información disponibles para todas las personas. El acceso abierto supone una vía, cada vez más rápida e inmediata, para garantizar la disponibilidad de la información y favorecer la visibilidad, uso e impacto de la investigación al favorecer la disposición de mayor cantidad de información científica sobre un área temática determinada, especialmente la localizada en las bases de datos más relevantes del área ${ }^{(1-4)}$.

El uso de las TIC es también imprescindible para asegurar el trabajo autónomo mediante la disposición de recursos digitales: campus virtuales, teleformación a través de gestores docentes virtuales, utilización de laboratorios virtuales y/o remotos, etc. En este marco, los estudiantes se enfrentan a nuevos retos pedagógicos, más orientados a la práctica, basados en la propia iniciativa, y en la adquisición de destrezas para las búsquedas, el tratamiento y la organización de la información científica disponible. En estos casos la docencia debe permitir a los estudiantes y futuros profesionales, desarrollar habilidades y estrategias para acceder, procesar y administrar la abundante información disponible, además de conocer las herramientas que se encuentran a su disposición, para que puedan ser competentes en la gestión de la producción científica sobre un área temática determinada ${ }^{(1,4,5)}$.

Los docentes tienen que enfrentarse a un nuevo proceso enseñanza- aprendizaje, con una relación pedagógica basada en la tutorización, utilizando más recursos didácticos, y adquiriendo mayores destrezas de "virtualización" $(1,4,5)$. La formación en el uso de la información implica el desarrollo de habilidades para un uso experto de los servicios bibliotecarios, y así optimizar resultados en el sistema educativo ${ }^{(6)}$. Los estudiantes son los protagonistas activos de su aprendizaje y tienen que adquirir una serie de habilidades que les permita afrontar las competencias específicas de sus áreas de conocimiento, ya que necesitan ser capaces de manejar el conocimiento, de actualizarlo, de seleccionar y conocer las fuentes de información y de comprender lo aprendido, para poderlo integrar en su base de conocimiento y que se puedan adaptar a las nuevas situaciones ${ }^{(1,7,8)}$. 
Como incipiente proceso de aprendizaje, se plantea la alfabetización en información donde se incluyen las tecnologías de la información y comunicación y la enseñanza elearning, todo ello centrado en la alfabetización digital. La alfabetización en información se entiende como el saber cuándo y por qué se necesita información, dónde encontrarla, cómo evaluarla, cómo utilizarla y cómo comunicarla de manera ética a los demás ${ }^{(7)}$.

La obtención de las habilidades para una alfabetización en información multiplica las posibilidades del estudio individual de los estudiantes, los compromete a utilizar una variedad de recursos de información para expandir sus conocimientos, hacer preguntas bien informadas y perfeccionar su pensamiento crítico y es la base para el aprendizaje a lo largo de toda su vida (lifetime learning) ${ }^{(1,2,9,10)}$. Las bibliotecas universitarias asumen una función constitutiva de su papel formativo en competencias informacionales. Por tanto, incorporar la alfabetización en información a los proyectos curriculares requiere de los esfuerzos conjuntos de docentes, bibliotecarios y administradores ${ }^{(9,11)}$.

Existen pocas investigaciones sobre habilidades de alfabetización en los estudiantes de Enfermería ${ }^{(12)}$. En una investigación llevada a cabo en los Estados Unidos de América (EE.UU.), se identificó que solo un 33\% de los coordinadores de los cursos de grado en Enfermería incluían en sus curriculums competencias en informática, tales como conocimiento sobre el uso de e-mail, procesamiento de texto, recuperación bibliográfica e internet ${ }^{(13)}$. Por otra parte, mientras que autores previos afirman que los estudiantes universitarios consideran que sus competencias sobre el uso de las TIC son bastantes elevadas, y se encuentran medianamente satisfechos con las condiciones de la docencia en las asignaturas en las que se está trabajando con el apoyo de las nuevas tecnologías ${ }^{(14)}$, en otros, los estudiantes de pregrado presentan conocimientos deficientes sobre los recursos que posee la informática, o en búsquedas bibliográficas, lo que impide emplear las TIC en las diferentes dimensiones en quée la Enfermería se relaciona, es decir, docencia, investigación, gestión y asistencia $^{(3,15)}$.

Teniendo en cuenta todo ello en un grupo de profesores de la asignatura Administración de Servicios de Enfermería del Grado de Enfermería de la Universidad de Sevilla se propuso desarrollar una experiencia para mejorar las destrezas de los estudiantes en alfabetización en información durante el curso 2009/2010. En este trabajo se presenta la experiencia, así como los resultados obtenidos de la evaluación de la misma.

\section{MATERIAL Y MÉTODOS}

\section{Desarrollo del proceso de enseñanza-aprendizaje}

La asignatura Administración de Servicios de Enfermería pertenecía a los estudios de Enfermería, en dicha asignatura estaban matriculados 93 estudiantes, de los cuales 80 estudiantes participaron en esta experiencia educativa, constituyendo un $86 \%$ del total.

El proceso enseñanza-aprendizaje de alfabetización en información se desarrolló en cinco sesiones de cuatro horas de duración cada una, siendo de 20 horas la duración total. Las dos primeras sesiones se impartían en las aulas de informática en grupos pequeños, la tercera sesión se realizaba de manera individualizada o en parejas y las 
sesiones cuarta y quinta eran para el trabajo autónomo o autogestionado del estudiante, fomentando su aprendizaje activo. Todas las sesiones eran supervisadas por un tutor/a.

La planificación y organización de los contenidos y de la evaluación del proceso enseñanza-aprendizaje se incluían en los proyectos docentes. Se siguió la siguiente organización, teniendo en cuenta la importancia de informar al alumnado en las principales bases de datos de interés en enfermería ${ }^{(16)}$ :

Sesión I: 1. Diseño de una estrategia de búsqueda; 2. Prácticas en bases de datos del organismo español CSIC (Consejo Superior de Investigaciones Científicas); 3. Presentación de bibliografías (APA, Vancouver, etc.) mediante el uso del RefWorks; 4. Prácticas de localización de revistas en el catálogo de la biblioteca de la Universidad de Sevilla "FAMA" y recursos electrónicos; 5. Manejo de las solicitudes de préstamo interbibliotecario.

Sesión II: 1. Prácticas sobre utilización del MESH DATABASE para guiar la búsqueda en PUBMED; 2. Prácticas en la base de datos de PUBMED; 3. Prácticas en la base de datos de SCOPUS, localizando las citaciones de las revistas y de los autores; 4. Prácticas en la Web of Knowledge (WOK), concretamente en Journal Citation Report (JCR) para conocer el factor de impacto y quartiles de las revistas; 5. Prácticas de traducciones al español de los abstracts.

Estas sesiones estaban apoyadas por tutoriales accesibles, a través de la plataforma de Enseñanza Virtual de la Asignatura, que incluían preguntas de elección múltiple para la autocomprobación de los conocimientos adquiridos.

Sesión III: En dicha sesión, en primer lugar los estudiantes seleccionaban un tema de su interés, relacionado con los contenidos del programa de la asignatura, sobre el que debían realizar una búsqueda bibliográfica (individualmente 0 en parejas) y entregaban, a la tutora, el titulo del tema elegido y las palabras claves, para que ésta aprobara su adecuación antes de que iniciaran la búsqueda. En segundo lugar, los estudiantes llevaban a cabo un aprendizaje sobre las características de la presentación y requisitos del informe del proceso de trabajo de alfabetización en información, es decir, de la búsqueda bibliográfica. En tercer lugar, se realizaba un control individualizado sobre los conocimientos adquiridos en el proceso enseñanzaaprendizaje en el aula de informática.

Sesiones IV y V: El propósito de estas sesiones tutoriales era promover el trabajo autónomo de los estudiantes en el manejo de la gestión de la información científica con el apoyo de la tutora. Se llevan a cabo mediante tutorías presenciales y/o no presenciales (enseñanza virtual y herramientas on-line).

El informe del proceso de trabajo de alfabetización en información que presentaban los estudiantes debía contener los siguientes elementos: 1) Los historiales de búsquedas de dos bases de datos (Index Medicus Español [IME] y PUBMED); 2) los registros seleccionados de un historial de cada una de las bases de datos utilizadas, junto con los resúmenes y las traducciones de los títulos y abstracts en el caso del PUBMED; 3) la bibliografía en estilo Vancouver utilizando Refworks; 4) los procedimientos llevados a cabo en la base de datos SCOPUS para conocer los datos de las citaciones de las revistas y de los autores/as; 5) el factor de impacto y quartil de una revista en la base de datos JCR; y 6) un artículo completo seleccionado por ser el 
más concordante, con el tema de búsqueda propuesto, de entre los historiales elegidos de las bases de datos IME y PUBMED.

\section{Evaluación de la experiencia}

Para la evaluación del informe elaborado por los estudiantes, durante el proceso de trabajo autónomo, se siguieron los criterios de calificación expuestos en la tabla 1. Se establecieron los siguientes rangos: menos de 0.7 puntos se consideró como suspenso; de 0.8 a 1 punto se consideró como aprobado; de 1.1 a 1.3 puntos se consideró como notable; y de 1.4 a 1.6 puntos se consideró como sobresaliente.

Tabla 1. Criterios de calificación de los informes. Elaboración propia. Criterios de calificación

- Presentación y adecuación de los historiales de búsqueda y registros con los resúmenes de los artículos del historial más adecuado al tema de búsqueda de IME y PUBMED

- Traducciones al español de los "abstracts" de PUBMED

- Presentación y adecuación de los procedimientos de búsquedas de la base de datos de SCOPUS y las citaciones de revista y autor/a

- Presentación de la bibliografía según Vancouver o APA, utilizando RefWorks

- Presentación del Factor de impacto ISI y el cuartil de la revista seleccionada

- Presentación de un artículo concordante con el tema de búsqueda seleccionado

Para la evaluación del proceso enseñanza-aprendizaje, se utilizó un diseño pretestpostest con un solo grupo experimental, al que se le aplicó un cuestionario de carácter anónimo y autoadministrado, antes y después del aprendizaje. Como variables demográficas se recogieron la edad, y el sexo/género. Se aplicó una escala de autovaloración del conocimiento, de tipo Likert, formada por seis ítems, con cinco opciones de respuesta (1.-totalmente de acuerdo, 2-de acuerdo, 3-indiferente, 4-en desacuerdo y 5-totalmente en desacuerdo). Dicha escala obtuvo valores Alfa de Cronbach óptimos en el pretest $\alpha=0.76$ y en el postest 0.78 . En el postest se introdujeron otras dos preguntas sobre la adecuación del aprendizaje y la satisfacción de los/as estudiantes con el mismo (Tabla 2).

Tabla 2. Ítems que componen el pretest y postest administrado a los/as estudiantes de Enfermería. Elaboración propia.

\begin{tabular}{ll}
\hline \multicolumn{1}{c}{ Ítems Pretest } & \multicolumn{1}{c}{ Ítems Postest } \\
\hline $\begin{array}{l}\text { 1.Tengo conocimientos suficientes para } \\
\text { buscar en la base de datos IME (CSIC) }\end{array}$ & $\begin{array}{l}\text { 1.Tengo conocimientos suficientes para } \\
\text { buscar en la base de datos IME (CSIC) }\end{array}$ \\
$\begin{array}{ll}\text { 2. Tengo conocimientos suficientes para } \\
\text { buscar en la base de datos PUBMED }\end{array}$ & $\begin{array}{l}\text { 2.Tengo conocimientos suficientes para } \\
\text { buscar en la base de datos PUBMED }\end{array}$ \\
$\begin{array}{ll}\text { 3. Tengo conocimientos suficientes para } \\
\text { buscar en la base de datos SCOPUS }\end{array}$ & $\begin{array}{l}\text { 3. Tengo conocimientos suficientes para } \\
\text { buscar en la base de datos SCOPUS }\end{array}$ \\
$\begin{array}{ll}\text { 4.Tengo conocimientos suficientes para } \\
\text { buscar las citaciones de los autores/as y } \\
\text { de las revistas científicas en la base de } \\
\text { datos SCOPUS }\end{array}$ & $\begin{array}{l}\text { 4.Tengo conocimientos suficientes para } \\
\text { buscar las citaciones de los autores/as y } \\
\text { de las revistas científicas en la base de } \\
\text { datos SCOPUS }\end{array}$
\end{tabular}


5. Tengo conocimientos suficientes para buscar el factor de impacto de las revistas científicas en la base de datos JCR

6. Tengo conocimientos suficientes para buscar información científica en general
5.Tengo conocimientos suficientes para buscar el factor de impacto de las revistas científicas en la base de datos JCR

6.Tengo conocimientos suficientes para buscar información científica en general

7.El aprendizaje teórico práctico en grupos pequeños en el aula de informática ha sido adecuado

8.En general estoy satisfecho/a con las prácticas de búsquedas de información

Se realizó un análisis descriptivo de las variables de estudio. Dado que los ítems del cuestionario estaban medidos en una escala ordinal, y mostraron una distribución distinta a la normal en las variables dependientes en la muestra, es decir, en los distintos ítems del cuestionario en el pretest y en el postest, así como en la escala completa de autovaloración del conocimiento, se ha aplicado la prueba no paramétrica $\mathrm{W}$ de Wilcoxon, para contrastar la hipótesis sobre la existencia de diferencias entre las medias de las respuestas a la escala de conocimiento entre el pre-postest, con un intervalo de confianza del 95\% ( $p<.05)$. La comprobación de si la muestra seguía una distribución normal, se realizó mediante la aplicación de la prueba Z de Kolmogorov-Smirnov, aceptándose la hipótesis alternativa, es decir, la distribución es diferente a la normal, en el caso de que la misma fuera significativa con un intervalo de confianza del $95 \%(p<.05)$ (Tabla 3). Se midió el tamaño del efecto, mediante el cálculo de la diferencia de medias estandarizadas (d) para los siguientes valores: $b a j o=0.2$, medio $=0.5$, alto $=0.8$. Se utilizó el programa estadístico SPSS 18.0.

Tabla 3. Aplicación de la prueba de Kolmogorov-Smirnov a la escala completa de autovaloración de conocimientos, en el pre y post exposición. Elaboración propia

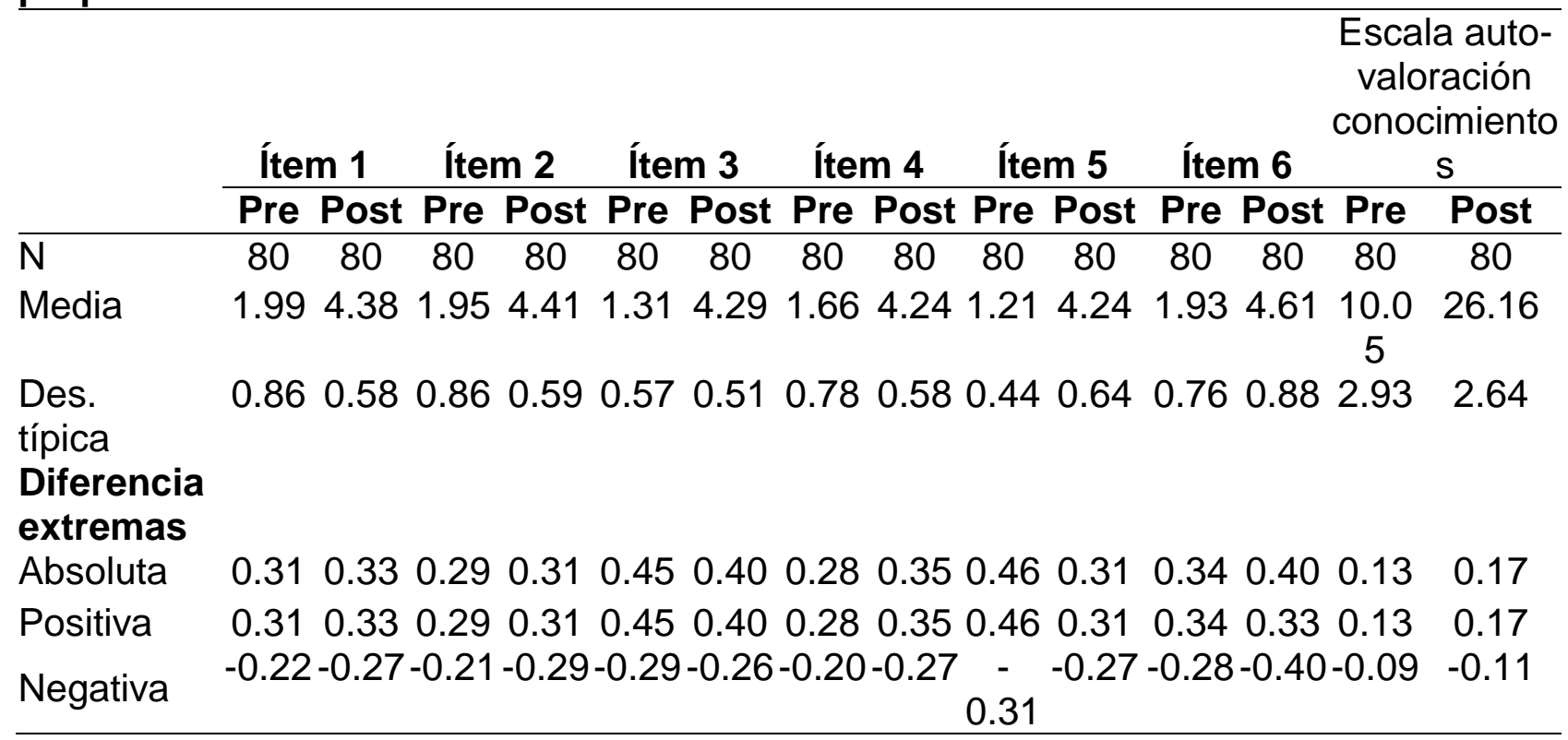


\begin{tabular}{lllllllllllllll}
\hline de Kol.- & 2.74 & 2.93 & 2.59 & 2.76 & 4.00 & 3.59 & 2.48 & 3.10 & 4.11 & 2.75 & 3.00 & 3.54 & 1.18 & 1.52
\end{tabular} Smirnov

Sig. asintót. .000 $000 \quad .000 .000 .000 .000 \quad 000.000 \quad 000.000 \quad .000 .000 .124 \quad .020$ (bilateral)

El estudio ha sido aprobado por el Comité Ético de Experimentación de la Universidad de Sevilla, habiendo solicitado el consentimiento informado a los/as estudiantes participantes.

\section{RESULTADOS}

La edad de los estudiantes osciló entre 20 y 37 años, siendo el promedio de 22.5 (21.8-23.3). El 73.70\% de los estudiantes eran mujeres y el 34 (26.30\%) hombres.

Resultados de los informes de búsquedas de información científica.

Se realizaron 49 informes, 32 de ellos por parejas, y el resto de manera individual. La mayoría de los estudiantes obtuvo la calificación de aprobado en el informe (35\%), seguidos de los que obtuvieron una calificación de notable (32.5\%), suspensos $(17,5 \%)$ y sobresalientes $(15 \%)$.

Resultados de la evaluación del proceso enseñanza-aprendizaje.

Se encontraron diferencias estadísticamente significativas entre las medias obtenidas en el pretest y el postest con respecto al grado de acuerdo de los/as estudiantes con cada uno de los ítems, así como en la escala completa de autovaloración de los conocimientos, pudiendo afirmar con un intervalo de confianza del $99 \%$ que dichas puntuaciones mejoraron significativamente tras la aplicación del proceso de enseñanza aprendizaje, siendo el tamaño de efecto muy alto en todos los casos. Asimismo, las puntuaciones medias obtenidas en las preguntas sobre la adecuación del aprendizaje y la satisfacción de los estudiantes con el mismos fueron muy elevadas. (Tabla 4).

Tabla 4. Diferencias obtenidas la escala completa de autovaloración de conocimientos, pre y post exposición al proceso de enseñanza-aprendizaje. Elaboración propia.

Ítems

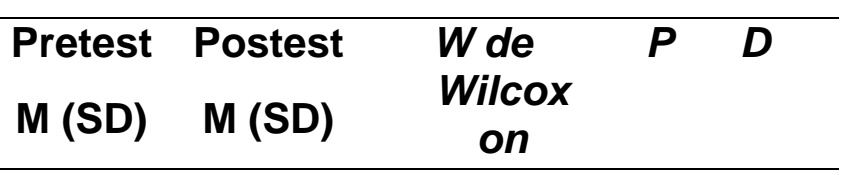

1. Tengo conocimientos suficientes para buscar en la base de datos IME (CSIC)

2. Tengo conocimientos suficientes para buscar en la base de datos PUBMED

3. Tengo conocimientos suficientes para buscar en la base de datos SCOPUS

4. Tengo conocimientos suficientes para buscar las citaciones de los autores/as y de las revistas científicas en la base de datos SCOPUS

5. Tengo conocimientos suficientes para

$\begin{array}{lllll}1.99 & 4.38 & -7.61 & .000 & 3.26 \\ (0.86) & (0.58) & & & \\ 1.95 & 4.41 & -7.66 & .000 & 3.34 \\ (0.86) & (0.59) & & & \\ 1.31 & 4.29 & -7.91 & .000 & 5.56 \\ (0.56) & (0.51) & & & \\ 1.66 & 4.24 & -7,71 & .000 & 3.75 \\ (0.78) & (0.58) & & & \\ 1.21 & 4.24 & -7.85 & .000 & 5.52\end{array}$


buscar el factor de impacto de las

revistas científicas en la base de

(0.44) (0.64)

datos JCR

6. Tengo conocimientos suficientes para buscar información científica en general

$1.93 \quad 4.61$

$(0.76)$

$(0.88)$

$-7.62$

$.000 \quad 3.26$

Puntuación total Escala de

Autovaloración de los Conocimientos en Alfabetización en Información

$10.05 \quad 26.16$

$(2.93 \quad(2.64) \quad-7,78 \quad .000 \quad 5.78$

7. El aprendizaje teórico práctico en

grupos pequeños en el aula de

informática ha sido adecuado

4.68

$(0.47)$

8. En general estoy satisfecho/a con las prácticas de búsquedas de información

4.6

En el pretest la mayoría de los/as estudiantes mostraron su desacuerdo con tener conocimientos sobre las herramientas propuestas (IME, PUBMED, SCOPUS), así como para la búsqueda bibliográfica en general. En el postest la mayoría de los estudiantes mostraron su acuerdo con tener conocimientos sobre estos aspectos. Prácticamente la totalidad de los estudiantes mostró su acuerdo con la adecuación y satisfacción con el proceso de aprendizaje (Tabla 5).

Tabla 5. Resultados de las respuestas de los ítems del pretest y el postest. Porcentajes y frecuencias absolutas. Elaboración propia.

\begin{tabular}{llll}
\hline Ítems & Pretest & Postest \\
& Desacuerdo Indiferente Acuerdo Desacuerdo & Indiferente Acuerdo \\
\hline
\end{tabular}

1. Tengo conocimientos

suficientes para buscar en la base de $81.3 \%$

datos IME (CSIC)

2. Tengo conocimientos

suficientes para buscar en la base de datos

PUBMED

3. Tengo conocimientos suficientes para buscar en la base de datos SCOPUS

4. Tengo conocimientos suficientes para buscar

$81.3 \%$

(65)

$95 \%$

(76)

$91.3 \%$

(73)
$5 \%$

(4)

(8)

$8.8 \%$

(7)

1.3

(1)

1.3

(1)

$97.6 \%$

(78) 
las citaciones

de los

autores/as y

de las revistas

científicas en

la base de

datos

SCOPUS

5. Tengo

conocimientos

suficientes

para buscar el

factor de

impacto de

las revistas

científicas en

la base de

datos JCR

6. Tengo

conocimientos

suficientes

para buscar

información

científica en

general

7. El

aprendizaje

teórico

práctico en

grupos

pequeños en

el aula de

informática ha

sido

adecuado

8. En general

estoy

satisfecho/a

con las

prácticas de

búsquedas de

información
$98.8 \%$

(79)

0

0

1.3

(1)

$7.5 \%$

(6)

$92.5 \%$

(74)

$87.6 \%$

(70)

$6.3 \%$

(5)

$6.3 \%$

(5)

0

1.3

(1)

$98.8 \%$

(79)

0

0

$100 \%$

(80)

1.3

(1)

$98.8 \%$

(79)

\section{DISCUSIÓN}

La mayoría de los estudiantes (82.5\%) superó la prueba final del proceso de enseñanza-aprendizaje. Además, el $47.5 \%$ de los estudiantes obtuvo puntuaciones altas (notable y sobresaliente) en el informe realizado, estos resultados concuerdan con los obtenidos en estudios donde se promueve el aprendizaje autónomo del estudiante $^{(10,15)}$. 
En el pretest administrado a los estudiantes de Enfermería, éstos mostraron un elevado grado de acuerdo respecto a los escasos conocimientos para hacer búsquedas de información científica. En estudios previos se afirma que los estudiantes de Enfermería autovaloran sus conocimientos sobre búsqueda de información de forma deficitaria ${ }^{(3,12,17)}$.

La autovaloración del conocimiento sobre alfabetización en información por parte de los estudiantes mejoró significativamente del pretest al postest en todos los ítems. Tras la realización del proceso enseñanza-aprendizaje, la autovaloración en cada uno de los ítems de la escala propuesta, así como el conjunto de la misma, aumenta de dos a tres puntos con niveles de significación $p<0.01$. La magnitud del efecto con valores $d>3.0$ resultó muy elevada, si consideramos los valores de referencias.

Investigaciones previas conducidas para evaluar cursos de alfabetización de la información para estudiantes de Enfermería demostraron diferencias significativas pre y post-programa en los conocimientos y habilidades respecto a este tópico. Así, se han hallado mayores niveles de autoconfianza en el uso de herramientas para la búsqueda de información, en estudiantes de Enfermería que realizaron un programa de alfabetización en información en comparación con un grupo control ${ }^{(18)}$ o importantes mejoras en los objetivos propuestos para la autovaloración de las destrezas en búsqueda de información, y en comparación con un grupo control ${ }^{(19)}$. Un programa de alfabetización en información dirigido a enfermeras postgraduadas, supuso un aumento del $50 \%$ en la autovaloración en el dominio de todas las competencias de alfabetización informacional por parte de los estudiantes, que identificaron el uso de las bases de datos electrónicas como la competencia más útil aprendida en el programa ${ }^{(20)}$. En otro estudio de cohortes para evaluar un programa de alfabetización en información en enfermeras postgraduadas, se encontraron diferencias estadísticas en la autoevaluación de las competencias en la cohorte que recibió el programa, con un elevado tamaño del efecto ${ }^{(12)}$.

La búsqueda de información científica es un importante componente de la alfabetización de la información. Las mejoras obtenidas en el postest en la autovaloración del conocimiento, junto a las capacidades demostradas en la realización de los informes para la búsqueda, la evaluación y la utilización de la información, pueden ser muestras de la adquisición de destrezas en alfabetización de la información por parte de los estudiantes tras completar el proceso de enseñanzaaprendizaje $^{(7,10)}$.

Con este intervención se ha contribuido, por tanto, al manejo de instrumentos y contenidos digitales, como las bases de datos de información científica, mediante la adquisición de las competencias adecuadas, siendo estas competencias relevantes en la formación continua de las personas a lo largo de toda la vida $(3,6,10,12,19)$. Además, la exposición de los estudiantes de Enfermería a estas herramientas puede tener gran impacto en los cuidados de salud, al facilitar que una vez estén graduados desarrollen una práctica basada en la evidencia, en la seguridad clínica y la toma de decisiones basada en la investigación científica ${ }^{(9,18,19)}$.

Por otra parte, el modo en que se ha realizado el entrenamiento puede haber facilitado el aprendizaje autónomo en los estudiantes, ya que al usar una amplia variedad de fuentes de información expanden sus conocimiento, aprenden destrezas para elaborar preguntas de investigación, y aumentan su capacidad para el pensamiento crítico en futuros aprendizajes ${ }^{\left(7,{ }^{10}\right.}$. Esto hace que la alfabetización de 
la información suponga una competencia importante para un proceso de enseñanzaaprendizaje eficaz ${ }^{(2)}$.

El proceso de aprendizaje reflexivo, autodirigido por el/la alumno/a, concordante con las temáticas impartidas en el mismo y apoyado en tutorías presenciales y virtuales, ha servido para reforzar y autocomprobar los conocimientos adquiridos que, en contraste con la enseñanza de técnicas de búsqueda de información de forma aislada, ha podido favorecer la adquisición de competencias y destrezas en alfabetización de la información, y la satisfacción de los estudiantes con la metodología utilizada ${ }^{(2,4,9,10,21)}$.

Investigaciones previas han señalado que la mayoría de las facultades de Enfermería no tienen programas adecuados sobre alfabetización de la información en sus asignaturas $(12,13,19)$, planteando otros autores que es necesario llevar a cabo investigaciones que den respuesta a esta problemática, por lo que la incorporación del aprendizaje en alfabetización de la información dentro del currículum de la titulación de Enfermería, puede asegurar que todos los estudiantes terminen sus estudios con altos niveles de esas competencias ${ }^{(1,10,13,16)}$.

Este estudio tiene algunas limitaciones. En primer lugar, se utiliza un diseño cuasiexperimental con un solo grupo, también llamado estudio antes y después, por lo que la ausencia de un grupo control no permite asegurar que la respuesta observada se deba exclusivamente a la intervención de estudio, ya que pueden haber influido otros factores no controlados ${ }^{(22)}$. Sin embargo, se consideró éticamente incorrecto dejar de administrar la intervención a parte de los alumnos matriculados. En segundo lugar, la escala utilizada realiza una autovaloración del conocimiento por parte del estudiante, pero no comprueba los conocimientos reales ni otros aspectos relacionados con las competencias, como son las actitudes o las habilidades. Es posible que aunque los estudiantes autovaloren sus conocimientos sobre búsquedas de información como elevados, existan variaciones con las competencias reales de los mismos. Otro potencial problema de la autovaloración por parte de éstos es que la baja valoración de conocimientos mostrada en el pretest puede indicar su falta de confianza en el uso de estas herramientas ${ }^{(13)}$. Sin embargo, el empleo de estrategias como el trabajo o informe de búsqueda de información científica permiten validar los resultados obtenidos en la escala, y pueden ayudar a demostrar la competencia del estudiante en alfabetización de la información.

\section{CONCLUSIONES}

Para que los estudiantes de Enfermería puedan hacer una buena gestión de la información, es necesario que consigan habilidades de alfabetización en información, mediante un aprendizaje basado en el trabajo autónomo y autodirigido, incluyendo la planificación, organización y evaluación de los contenidos en los proyectos docentes de las asignaturas. Todo ello fomenta un aprendizaje reflexivo y significativo y constituye un mecanismo de feed-back para el proceso de aprendizaje del/la estudiante.

Es imprescindible fomentar el aprendizaje activo y colaborativo de los estudiantes, el apoyo en las nuevas tecnologías como los campus virtuales y el interés por la actualización de los conocimientos a lo largo del periodo de formación y del posterior desarrollo futuro profesional basado en la investigación y en la evidencia científica. 


\section{BIBLIOGRAFÍA}

1. Button D, Harrington A, Belan I. E-learning \& information communication technology (ICT) in nursing education: A review of the literature. Nurse Educ Today. In press [acceso 10 Septiembre 2013]. pii: S0260-6917(13)00165-2. doi: 10.1016/j.nedt.2013.05.002.

2. Veiga J; Martín-Rodero, H. Acceso Abierto: nuevos modelos de edición científica en entornos web 2.0. Salud Colectiva. 2011; 7(1), S19-S27.

3. Huamani-Navarro M, Alegría-Delgado D, López-Sánchez M, Tarqui-Mamani CB., Ormeño-Caisafana L. Conocimientos, practicas y habilidades sobre la búsqueda bibliográfica y percepción estudiantil sobre la capacitación universitaria en investigación, en estudiantes de obstetricia. Educ Med. 2011; 14 (4): 235-2402.

4. López-Morales M, Celma-Vicente M, Cano-Caballero M. Dolores, Quero-Rufián A, Rodríguez-López MA. Docencia universitaria semipresencial. Experiencia en el uso de la plataforma virtual SWAD. Educ Med 2011; 14 (4): 229-234

5. San Miguel B. Las tecnologías de la información y la comunicación en el Espacio Europeo de Educación Superior [Editorial]. Salud Colectiva. 2011; 7(1), S5-S7.

6. Caridad M, Marzal MA. Políticas de información y alfabetización en información como medios de la inclusión social desde la óptica europea. Inclusão Social. 2006; 1(2): 31-43.

7. Pinto M. Habilidades y competencias de gestión de información para aprender a aprender en el Marco del Espacio Europeo de Enseñanza Superior [internet]. PORTAL ALFIN- EEES 2005 [acceso 10 Septiembre 2013]. Disponible en: http://www.mariapinto.es/alfineees

8. Daniele V, Rino MM. Currículo por competencia y metodología activa: percepción de estudiantes de enfermería. Rev. Latino-Am de Enfermagem, 2010 [acceso 10 Septiembre 2013]; 18(1). Disponible en: http://www.scielo.br/pdf/rlae/v18n1/es_17.pdf

9. Duquesne A. La alfabetización en información en los Policlínicos Universitarios. Educación Médica Superior 2011; 25(2):157-63.

10. Barnard A, Nash R, O’brien M. Information Literacy: Developing lifelong skills thought Nursing Education. J Nurs Educ. 2005; 44 (11), 505-510.

11. Uribe-Tirado A, Girlesa A. La alfabetización informacional en las universidades españolas. Niveles de incorporación a partir de la información publicada en los sitios web de sus bibliotecas-CRAI. Rev Esp Doc Cient. 2012; 35 (2): 325-345.

12. Tarrant M, Dodgson JE, Law BVKK. A curricular approach to improve the information literacy and academic writing skills of part-time post-registration nursing students in Hong Kong. Nurse Educ Today. 2008; 28: 458-68.

13. Mcneil BJ, Elfrink VL, Bickford CJ, Pierce ST, Beyea SC, Averill C, et al. Nursing information technology knowledge, skills, and preparation of student nurses, nursing faculty, and clinicians: a U.S. Survey. J Nurs Educ. 2003; 42(8): 341-349.

14. Silva ISA, Marques IR. Conhecimento e barreiras na utilização dos recursos da Tecnologia da Informação e Comunicação por docentes de enfermagem. J Health Inform. 2011; 3(1): 3-8.

15. García-Valcárcel A.; Tejedor-Tejedor F. Variables TIC vinculadas a la generación de nuevos escenarios de aprendizaje en la enseñanza universitaria. Aportes de las curvas ROC para el análisis de diferencias. Educación XX1. 2011; 14: 43-78.

16. Jiménez JA, Guillén JF, Murillo R. Fuentes de documentación para enfermería. Enfermería Global. 2006 [acceso 10 Septiembre 2013]; 5(1). Disponible en: http://revistas.um.es/eglobal/issue/view/63

17. Jacobsen HE, Andanaes R. Third year nursing students' understanding of how to find and evaluate information from bibliographic databases and Internet sites. Nurse Educ Today. 2011; 31: 898-903. 
18. Shorten A, Wallace MC, Crookes PA. Developing information literacy: a key to evidence-based nursing. Int Nurs Rev. 2001; 48(2): 86-92.

19. Wallace MC, Shorten A, Crookes PA. Teaching information literacy skills: an evaluation. Nurse Educ Today. 2000; 20: 485-489.

20. Jacobs SK, Rosenfeld PH. Information literacy as the foundation for evidencebased practice in graduate nursing education: a curriculum-integrated approach. $J$ Prof Nurs. 2003; 19(5): 320-328.

21. Schutt MA, Hightower B. Enhancing RN-to-BSN students' information literacy skills through the use of instructional technology. J Nurs Educ. 2009; 48(2): 101-105.

22. Argimon JM, Jiménez J. Métodos de investigación clínica y epidemiológica. $3^{\text {a }}$ Ed. Barcelona: Elsevier; 2000. 